This item was submitted to Loughborough's Research Repository by the author.

Items in Figshare are protected by copyright, with all rights reserved, unless otherwise indicated.

\title{
Optimization of assembly instructions for a low-cost housing solution
}

\section{PLEASE CITE THE PUBLISHED VERSION}

https://benjamins.com/\#catalog/journals/idj.22.1.04tor/details

\section{PUBLISHER}

(C) John Benjamins Publishing Company

\section{VERSION}

AM (Accepted Manuscript)

\section{LICENCE}

CC BY-NC-ND 4.0

\section{REPOSITORY RECORD}

Torres-Sanchez, Carmen, Changxi Huang, and Garry Steel. 2019. "Optimization of Assembly Instructions for a Low-cost Housing Solution”. figshare. https://hdl.handle.net/2134/21378. 


\section{Optimization of assembly instructions for a low- cost housing solution}

Bamboo huts have been proposed as a low-cost housing solution in places like India, the Far East and South America. The assembly of these huts is a task for the end-user in an attempt to empower communities. Successful building is strongly linked to the end-user's ability to interpret and execute the assembly instructions correctly. This relies on the quality of the planning and presentation of the guiding instructions. Because the end-user is an audience with a broad spectrum of literacy, competences and capabilities, these instructions have to be carefully prepared and illustrated. In this work, the planning of the structure instructions was carried out with the overall aim to decrease complexity and increase effectiveness so that the assembly could be interpreted and executed correctly. Best practices for the presentation of the assembly instructions (i.e., creation of layout and diagrams) reported in the literature were observed. A diagnostic test to assess their suitability was conducted. This allowed the improvements in intelligibility and clarity in the interpretation of those assembly instructions. Attending to organizational-dynamic sequencing, cultural relevance, as well as skillset and technical background of the participants, the results provided insight into the way in which end-users dealt with ambiguity and intrinsic cognitive load. An optimized version of the instructions reflected the compiled preferences for sub-assemblies, action and colored diagrams with operations that decrease cognitive load (e.g., labelling, magnified details), and self-auditing steps. Concrete recommendations can be distilled from this case study and are reported for the benefit of the instructional designer.

Keywords - Bamboo, instructions, cognitive load, sequence, diagnostic testing.

\section{Introduction}

Bamboo has long been used as a feasible and sustainable solution for building low-cost structures in developing countries in Africa, South America, Asia and the Far East (Janssen, 1995). As a grass of remarkable mechanical properties, its widespread availability and rapid growth have made it an interesting structural material due to its affordability, easy assembly 
and relatively long durability (Minke, 2012). In the Far East bamboo is widely used in scaffolding structures (Chung \& Yu, 2002). In India bamboo is seen as a solution to housing and storage of goods and livestock by those who have little. Concrete and steel structures for buildings are typically costly, difficult to prepare and assemble, requiring skilled workers and quality materials for any truly successful result. Bamboo offers an easier alternative (Mishra et al., 2009) (Figure 1). The deployment of bamboo frameworks that can be easily assembled for dwelling, commercial or civil use and in disaster relief campaigns has been reported (Leake et al., 2010). Following up on this, Drake et al (Drake et al., 2011) implemented the use of low-cost bamboo structures in rural areas of India. One of the main outputs extracted from that fieldwork revealed the need for user-friendly and clear instructions to achieve a satisfactory assembly.
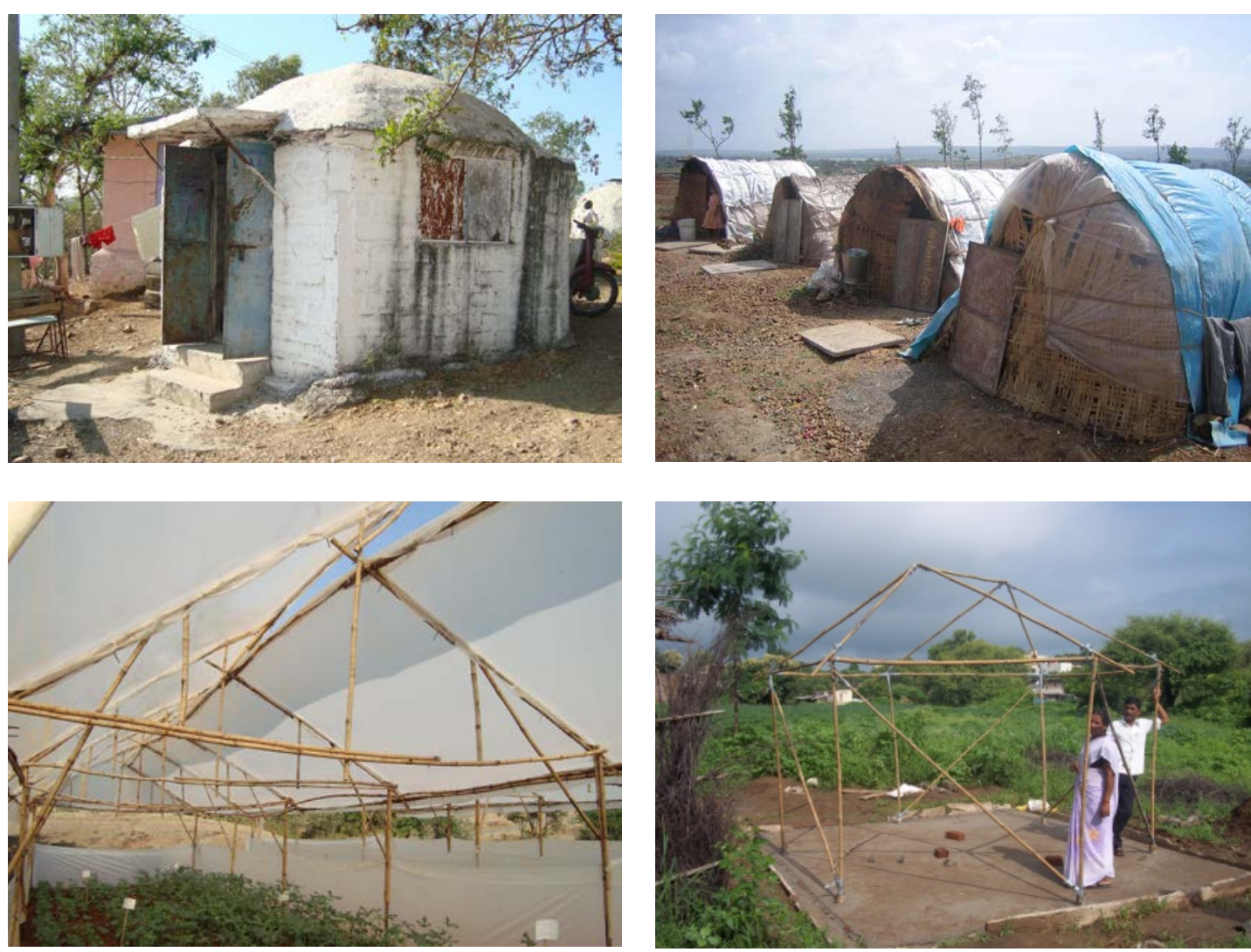

Figure 1: (top left) An expensive housing solution in India; (top right) A humble dwelling in India; (bottom left) An example of bamboo hut used for livestock and crops; (bottom right) construction of low-cost bamboo structures (Drake et al., 2011). These communities would benefit from intuitive and feasible instructions 
This paper presents an attempt to create an optimized set of intelligible instructions that allows the end-user a successful and quick construction of a bamboo framework hut. For this purpose, we draw upon the experience and research undertaken in the study of operations in assembly lines (e.g., mass manufacture of commodities) and the optimization of assembly instructions for ready-to-assemble products. The latter are of particular interest in this study. Ready-to-assemble items such as furniture, recreational equipment and toys have been very successful in selected markets (Bramorski et al., 2000). Consumer-oriented design has become a major thrust in the product development process (Hsieh et al., 2013) and this is particularly true in the Do-it-Yourself (DIY) arena. Most of that success stems from the fact that DIY products are specifically designed to allow the customer to assemble their products through the use of intuitive assembly instructions and commonly available tools. Even more, the success of a product relies on the end-user's ability to interpret and enact those instructions correctly (Schumacher, 2013). Understanding how people think about and interact with the process of assembling an object is at the core of the optimization of instructions. Consequently a structured design process for instructions (which includes a combination of algorithms and cognitive design principles) is crucial for the success of a product and its endorsement by end-users.

This paper is divided into four sections. Initially we report the foundations for the preparation of intelligible and effective assembly instructions based on literature on the matter. Principles for the planning and the presentation are outlined. Then the methodology used in this work for the testing of those principles is described. A compilation of the results is summarized before we draw conclusions and learnings from the process.

\section{Intelligible and effective assembly instructions}

There is a robust body of work around the activity of creating effective and intuitive assembly instructions for consumer products. Although this task is considered as single-use (i.e., the user is typically exposed to the task only once) and lacks an intentional learning aspect, recommendations and best practices in learning percolate indirectly the design decisions for the assembly instructions (Clark et al., 2011; Heiser et al., 2004; Sweller, 1994). Recommendations (Söderberg et al., 2014) for the development of such guidelines have guided this work in the two main stages involved in this process: 
1) Planning phase, that involves mapping of the assembly and planning of the instructions structure

2) Presentation phase, which relates to the creation of instructions layout, pictures and text, and their enhancement as the viewpoint of the user or the operator is captured via testing and interviews.

\section{Planning}

The mapping of the assembly was considered strongly dependant on the physical features (geometry, trusses) of the hut. Since these have an impact on cognition and instruction comprehension (Richardson et al., 2004), the socio-economic and cultural environment where the end-user would utilize this low-cost housing solution had to be taken into consideration. The end-users are also expected to be in charge of its assembly. They might be unfamiliar with assembly hut techniques, have no formal training or exposure to the pictorial conventions of technical communication and also be semi-literate. Statements and interviews with members of local communities typically help to extract product design specifications for the product and the participants' experiential opinions. This information was available from the low-cost housing fieldwork in India conducted previously (Drake et al., 2011). The observations in-situ were in agreement with the conclusions extracted from the surveying work on communication in developing countries (Chandler et al., 2014; Houts et al., 2006; Schumacher, 2009), and they were the reference points for the planning stages. In this work it was paramount to be attentive to end-user's necessities and to their attitudes towards the instructions. Their broad range of literacy levels as well as of skillsets for the manual work demanded a set of instructions that could cater to a wide audience, maintaining accessibility and most importantly, non-patronising. There is a widespread preconceived misconception about the developing world that corresponds to an misinformed and unglorified view of the level of literacy and capabilities of the people in those countries (Rosling et al., 2005). In the light of that, the end-user of the proposed bamboo hut was not seen as a passive character, a 'recipient of message', in contrast to the view of a so-called western expert who performs an evangelical function and sends one-way messages (Fuglesang, 1973). Consequently the planning of the instructions structure was guided by the following principles:

A. Pictorial-only representation was preferred to pictures and text 
The decoding of written instructions for an assembly requires mental transformation by the reader. In contrast, visuals shortcut that path (Ganier, 2004) and enhance elaboration of the mental model. Pictures and diagrams ease the process of building these mental models and are preferred for illiterate, semi-literate audiences and for those with a difficulty to read (Söderberg et al., 2014). The principal hurdle in the process of interpretation is the different levels of familiarity with the visual communication that each end-user has. We studied the work done on children experiences with pictorial representations (Lopes Peres \& Barreto Campello, 2014), the use of graphics in patient education, and storyboards in counselling (Houts et al., 2006). The findings extracted from those case studies informed our planning stage. Children develop at different rates and although they will interact differently with images, representations and sequential operations, they all have in common a large interest in illustrated instructions. When dealing with healthcare patients, pictures were seen to improve comprehension, and markedly increased attention and the eliciting of health education advice. Patients with low literacy skills were especially likely to benefit from visual media (Chandler et al., 2014). Based on those findings, we favorited the use of pictorial-only representations for our instructions in an attempt to reach a wider audience (from illiterate to literate), increase friendliness ('play’ versus ‘serious work') and familiarity.

\section{B. Cognitive load was kept low}

Authors who have worked on this topic have shown that cognitive load affects operator performance (Ganier, 2004; Richardson et al., 2004; Sweller, 1994). Studies from cognitive experiments aimed to reduce guesswork, free interpretation and prevent misuse of the product were recalled and enacted in this case. In an assembly situation the short-term memory is the working memory (Ganier, 2004). The cognitive load is reduced when the number of items to deal with mentally at one given time (e.g., number of new actions per sequential step) is large unless a good strategy for grouping them, or 'chunking' (Miller, 1956), is put in place for the benefit of the end user.

We intended low levels of abstraction for the representation of the instructions as the level of abstraction has an impact on the complexity of the task perceived by the user. This included the use of no more than six new items per step, the use of line diagrams with varying line widths to emphasize mass, proximity and scale and to differentiate between structural parts (thick line) and actions or labels (thin line), and of realistic perspective with stable points of view. High level abstraction techniques such as exploded views, cutaways, 
deformation and distortions (e.g., magic lenses, fish-eye views, etc) were avoided or used only sporadically and when not conflicting with the perspectives (Kim et al., 2010; Viola \& Groeller, 2005). We considered that introducing high levels of abstraction techniques could create misunderstanding and confusion. However, because these had to be used at times, the planning of the instructions required a careful mix of high-level with low-level abstraction items (e.g., keeping to strictly one high level abstraction resource per vignette), as suggested in reported best practices (Söderberg et al., 2014; Spinillo \& Dyson, 2001).

The cognitive load can also be lowered by presenting instructions that facilitate locating and comprehending the information that is to be implemented (e.g., using a linear organization of the operations sequence attending to the spatio-temporal nature of the pictures - which are a language of transformation when organized in a sequence (Lopes Peres \& Barreto Campello, 2014), color-coding the parts and/or the sequences, numbering the operational steps, increasing similarity of the structure via symmetry planes, etc. (Ganier, 2004). Grouping the component parts in a package according to the order of assembly also reduces difficulty in identifying the parts (Madan et al., 1995). The use of this strategy intended to be inclusive towards those less capable technically end-users not to feel overwhelmed but engaged in a task that is portrayed as accessible.

C. Simplification of the assembly complexity by identification of challenging operations and tasks in the instructions sequence

We employed hierarchical task analysis (HTA), a general framework for examining tasks in the context of operations (and sub-operations) and goals (with sub-goals) (Jonassen et al., 1999; Shepherd, 1998; Stanton, 2006). The assembly was decomposed into operations, tasks, actions and parts. These had an inherent hierarchical link so that parts were grouped by: (i) a single action. This is, parts in the same hierarchy were to be added at the same time-step and at a sequence (one after the other), for example, the vertical poles for the body of the hut; or (ii) form subassemblies, for example, the roof of the hut, which coincidentally could decrease the cognitive load. When we anticipated that certain operations and tasks would lead to assembly complexity, resources based on 'Task Operation - Task Evaluation’ (TOTE) such as 'check points' were introduced. Some of the TOTEs (Shepherd, 1998) relevant to this assembly exercise and utilised here were diagrams to show correct positioning, orientation and rotation of parts. These state-check points in the sequence aided the user to confirm that their understanding of the operation was correct, that they have executed the action 
successfully and the next step in the assembly could be attempted. Likewise, this strategy allowed for an enhanced accessibility to a wider audience.

\section{Presentation of the assembly instructions}

The framework for the presentation of the instructions was defined by the preparatory planning stage and the boundaries delimited by the on-location reality of the end-users. We observed best practice reported in literature and distilled from practices in the assembly of Do-It-Yourself (DIY) and flat-packed consumer products (Agrawala et al., 2003; Heiser et al., 2004; Heiser \& Tversky, 2006; Houts et al., 2006; Miller, 1956; Schumacher, 2007; Söderberg et al., 2014; Spinillo \& Dyson, 2001). Three aspects of instructions were surveyed: the quality of the Illustration, the Viewing Angle and the Action. A compilation of those is presented in table Table 1.

Table 1: Compilation of best practices for the presentation of effective instructions

\begin{tabular}{|c|c|c|}
\hline & & Action \\
\hline 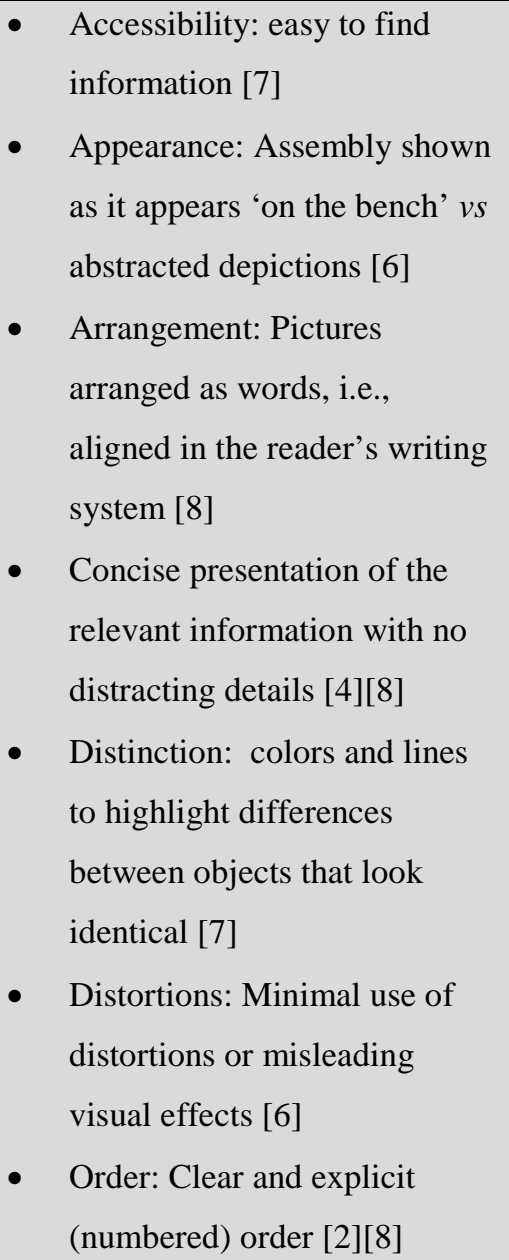 & 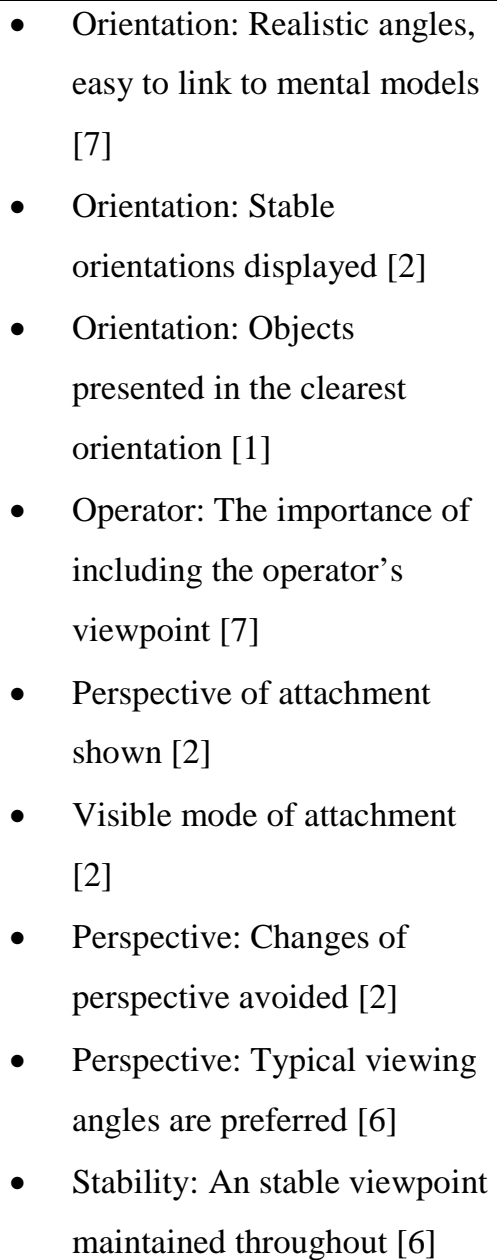 & 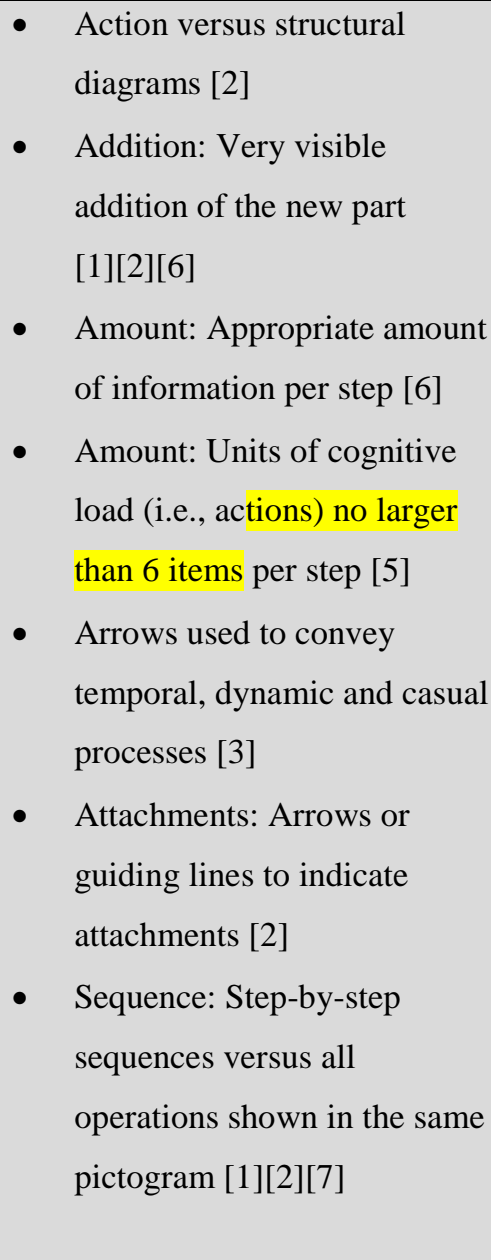 \\
\hline
\end{tabular}



[1](Agrawala et al., 2003)
[5] (Miller, 1956)
[2] (Heiser et al., 2004)
[6] (Schumacher, 2007)
[3] (Heiser \& Tversky, 2006)
[7] (Söderberg et al., 2014)
[4] (Houts et al., 2006)
[8] (Spinillo \& Dyson, 2001)

The guiding principles for the planning and the presentation of the instructions described in this section allowed the design of an initial set whose suitability was assessed by iterative diagnostic testing. Details on the iterations that yielded an optimum version are presented in the next section.

\section{Methodology and Testing}

Since the optimization of the hut assembly instructions can be considered mainly a faultfinding, iterative process, we chose the Diagnostic Testing technique for this purpose. The tests were designed with the aim to identify strengths and weaknesses in the instructions that aided or deterred user's interpretation, provide instant detailed feedback which could be acted upon, and therefore lead to remediation in the subsequent iteration of the instructions. As defined by Schumacher (Schumacher, 2007), diagnostic testing is "used to create and refine a specific design for a particular problem”. After each testing iteration feedback was collected, errors detected, refined new designs proposed and then retested in a new iteration. Because it is not a design methodology per-se, the number of selected participants was permitted to be low and these were those more likely to encounter problems when exposed to the instructions for the first time. Therefore difficulties with the design (e.g., ambiguity, inappropriate use of the parts, etc.) could be made evident and in that way cognitive complexity lowered for the following iteration.

We evolved the presentation of the assembly instructions from a series of three diagnostic tests that evaluated the instructions in four aspects: (i) organisational sequencing (i.e., hierarchical vs subassemblies presentation), (ii) dynamic sequencing (i.e., structural vs action diagrams), (iii) cultural relevance (incl. color-coded diagrams and subassemblies \& segmentation), and (iv) skillset relevance.

\section{Demographics of the participants}

26 people of age (21-49) participated in the experiments. 17 of them were male and 9 female. There were 10 European nationals (Scottish, English and Irish) and 16 Asians or Far East 
nationals (Chinese, Indian and Koreans). The number of participants with an engineering or technical background (in visualization or modelling) was 17, and 9 of them had no formal training in 3D representational conventions. Neither gender nor age-related performance variables were under study. All participants were literate.

\section{The diagnostic testing}

The experimental sessions were run indoors to avoid any distractions of the participants or interruptions due to weather conditions. The experimental rooms comprised a large open space surrounded by desks and chairs that the participants could use during their work. The hut consisted of structural parts (i.e., bamboo beams), metal connectors that position the beams, and fasteners (i.e., cable ties) to hold those together. These components were supplied along with the instruction set tailored for each exercise. The participants worked in teams of two. All participants were novices (i.e., they had not seen the instructions or were familiar with the assembly of the hut) at all stages of the iterative process (i.e. only experienced the activity once). At the start of each experiment the participants were briefed on health and safety issues, given the set of instructions (Figure 2) and left to familiarize themselves with the different parts and components which were displayed on the floor or on the desks (Figure 3). The length of time that the teams spent in the different activities (i.e., reading, building the body and the roof of the hut) was recorded (Figure 4). In a post-exercise interview the participants were asked to score the difficulty of each of the instruction steps using the (1-10) difficulty-easy scoring (Likert, 1932) (bottom of each diagram in Figure 2). The participants were requested to indicate the most useful step, task or operation, the most difficult ones to understand, and which parts were the most difficult to assemble. The participants were also requested to provide suggestions for improvement of the clarity of the instructions. Each assembled hut was scored in terms of stability (1-5, 1 being very unstable) and qualitatively assessed its resistance to tensile transversal force (yes/no). An inventory of the parts (bamboo poles, metal connections) not used in the suggested correct position was undertaken, as well as the amount of fasteners used by each group in their assembly exercise. 


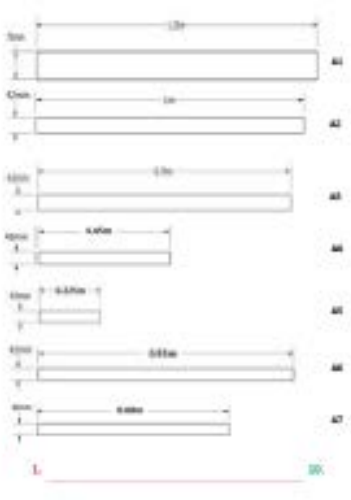

3.

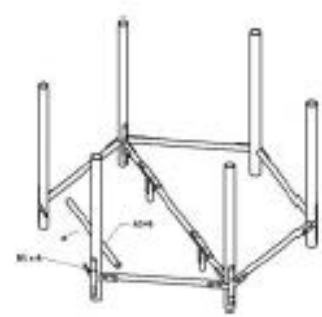

7.

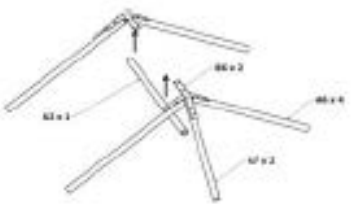

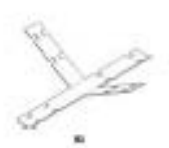
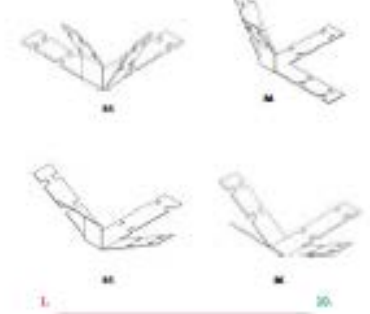

4.
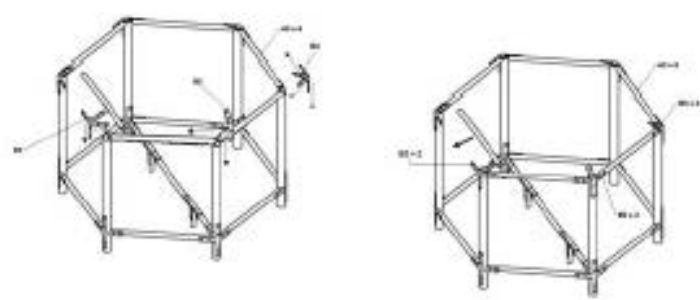

9.

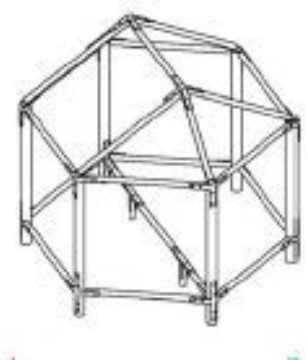

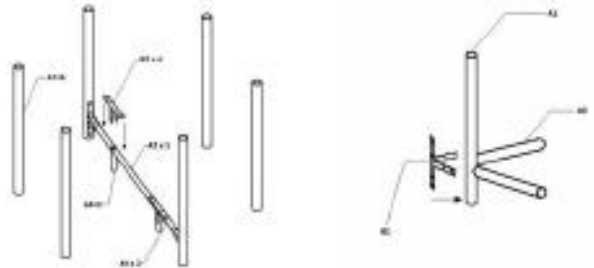

5.

6.

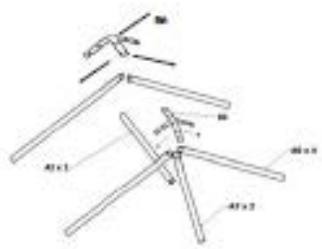

Figure 2: Example of set of instructions used during the diagnostic test 


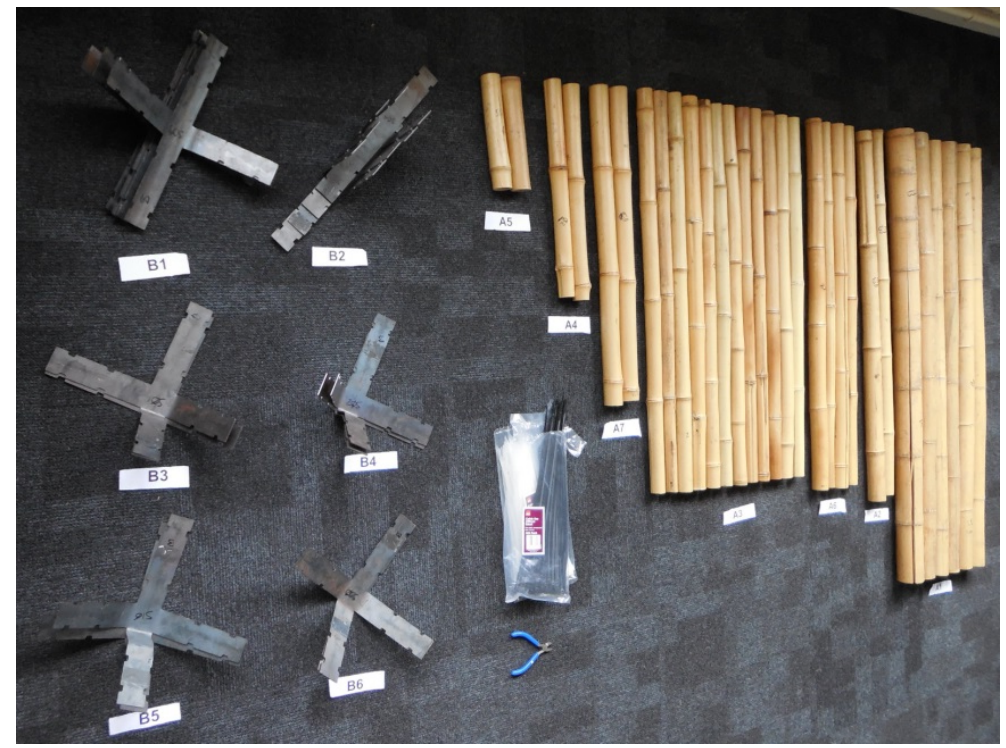

Figure 3: Parts and components as laid-out at the start of the experiments. User testing indicated that labelling was a strong feature towards feasibility of a rapid assembly
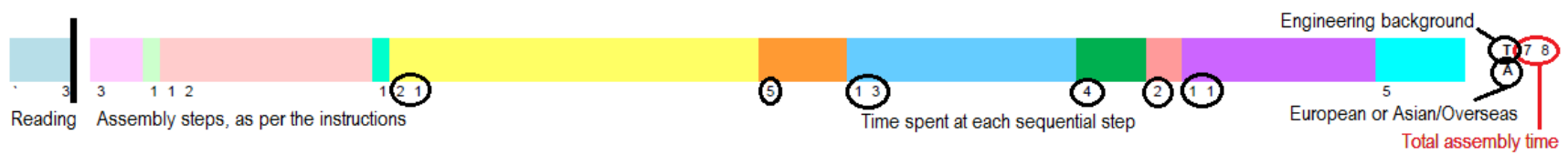

Figure 4: Exemplar of the timing of activities based on the set of instructions in iteration 2

\section{Results and Discussion}

\section{First Iteration}

The first iteration involved the study of sequencing in its organisational and dynamic nature:

(i) hierarchical versus sub-assembly presentation and (ii) structural versus action diagrams.

The instruction sets were black and white line diagrams. The participants were Europeans and had a technical background.

(i) Organisational sequencing: hierarchical vs sub-assembly presentation

The assembly task under study allowed a comparison between vertical assembly (i.e., bottom up, from floor to roof) and the creation of sub-assemblies that are put together to form the final hut (Figure 5). 
2.
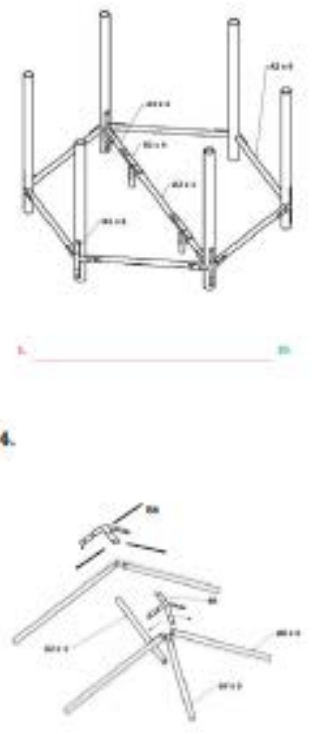
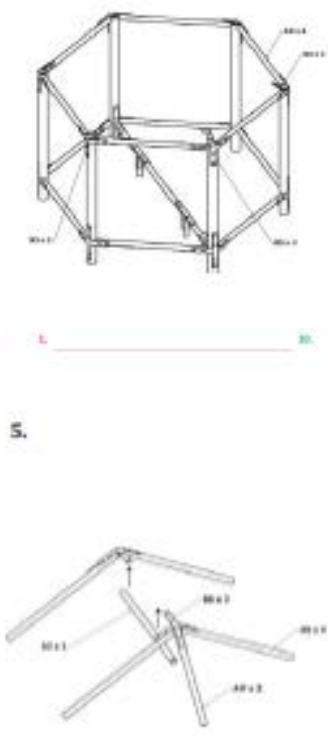

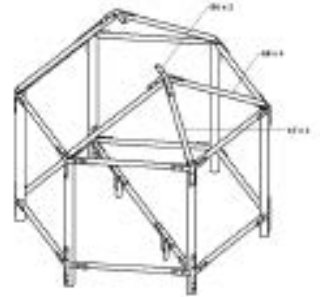

6.

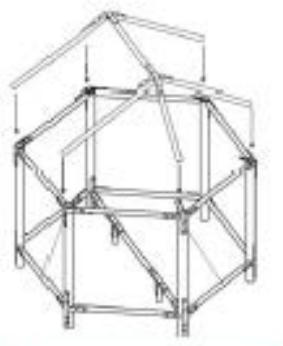

Figure 5: Exemplars of sequence for the hierarchical method (top row) and sub-assembly approach (bottom row). Participants preferred sub-assembly representations

Four groups were tested. Two of them were presented vertical assemblies and two were instructed to perform sub-assemblies. Sub-assemblies were spontaneously created even when the instructions explicitly stated to use the parts hierarchically. The groups which were suggested the sub-assemblies approach went beyond and created smaller sub-assemblies within the larger sub-assemblies. This observed approach comes to reinforce the preference for sub-assemblies in the object assembly operation. The completion time of the subassembly teams was shorter than that of the vertical assembly teams, and the former ones exhibited more engagement throughout the activity. All groups suggested the use of color in the schematics to improve clarity in the sequence.

(ii) Dynamic sequencing: static vs action diagrams.

The assembly instructions were designed to present a process of static diagrams or an action with arrows indicating the building sequence (Figure 6). The aim of a static diagram is to show the clearest of views on the different steps in the sequential operations. In contrast, the 
action diagrams show how to attach components (e.g., connections) to the structure. Arrows were chosen for the purpose of conveying change, movement and causality as a symbolic device familiar to the users (Heiser \& Tversky, 2006). The four groups were observed for understanding the preference for one or the other type of representation. The assembly depicting action diagrams was preferred to that of structural diagrams. Total assembly times were faster and the participants reported a lower level of confusion when it came to determining which parts to join and assemble. All groups suggested enlarging busy areas of the diagrams, and three of the groups the labelling of the components to ease the selection and orientation tasks. The use of color continued to be suggested as an enhancer for clarity.

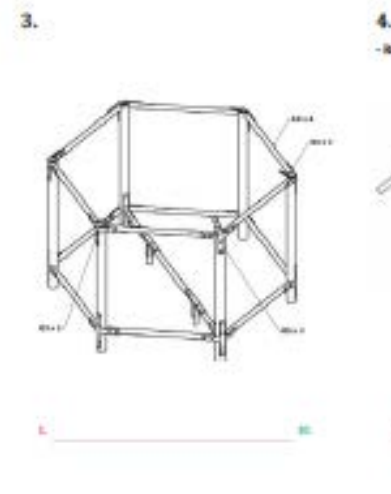

5

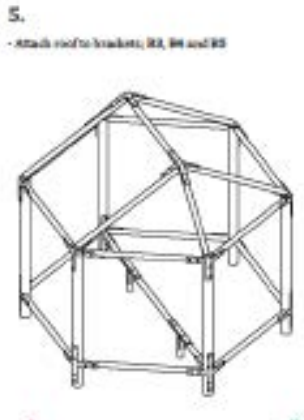

3.
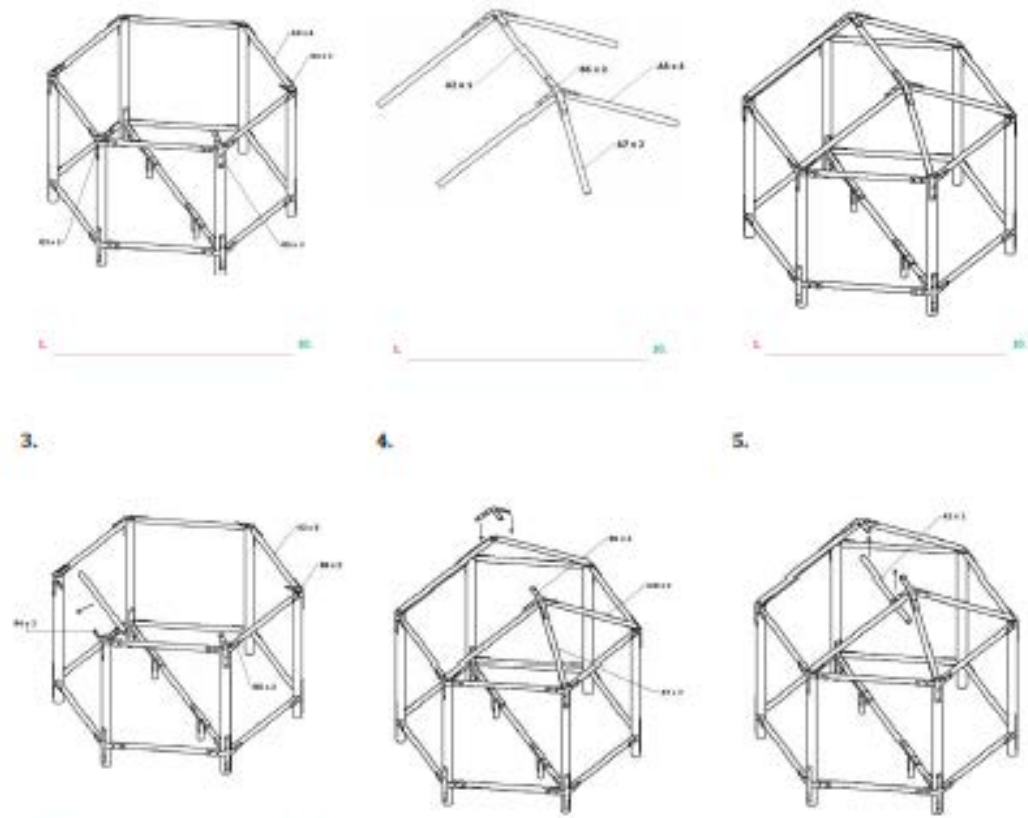

Figure 6: Exemplars of sequence for the static (top row) and action approaches (bottom row). Arrows indicating action were generally preferred for guidance

\section{Second Iteration}

The second iteration of the optimisation of the assembly instructions studied the improvements within the framework of cultural relevance. The new sets were designed to test on aspects such as (iii) use of color and (iv) sub-assemblies and segmentation. The participants were of Chinese and Indian nationality. All of them had a formal training in technical studies or engineering. 
The items under study were the use of color-coded parts in the diagrams. Parts of the same hierarchy (for example the vertical poles or the connections on the bottom plane) were identified by a unique color throughout the whole sequence, in contrast to black and white line diagrams (Figure 7).

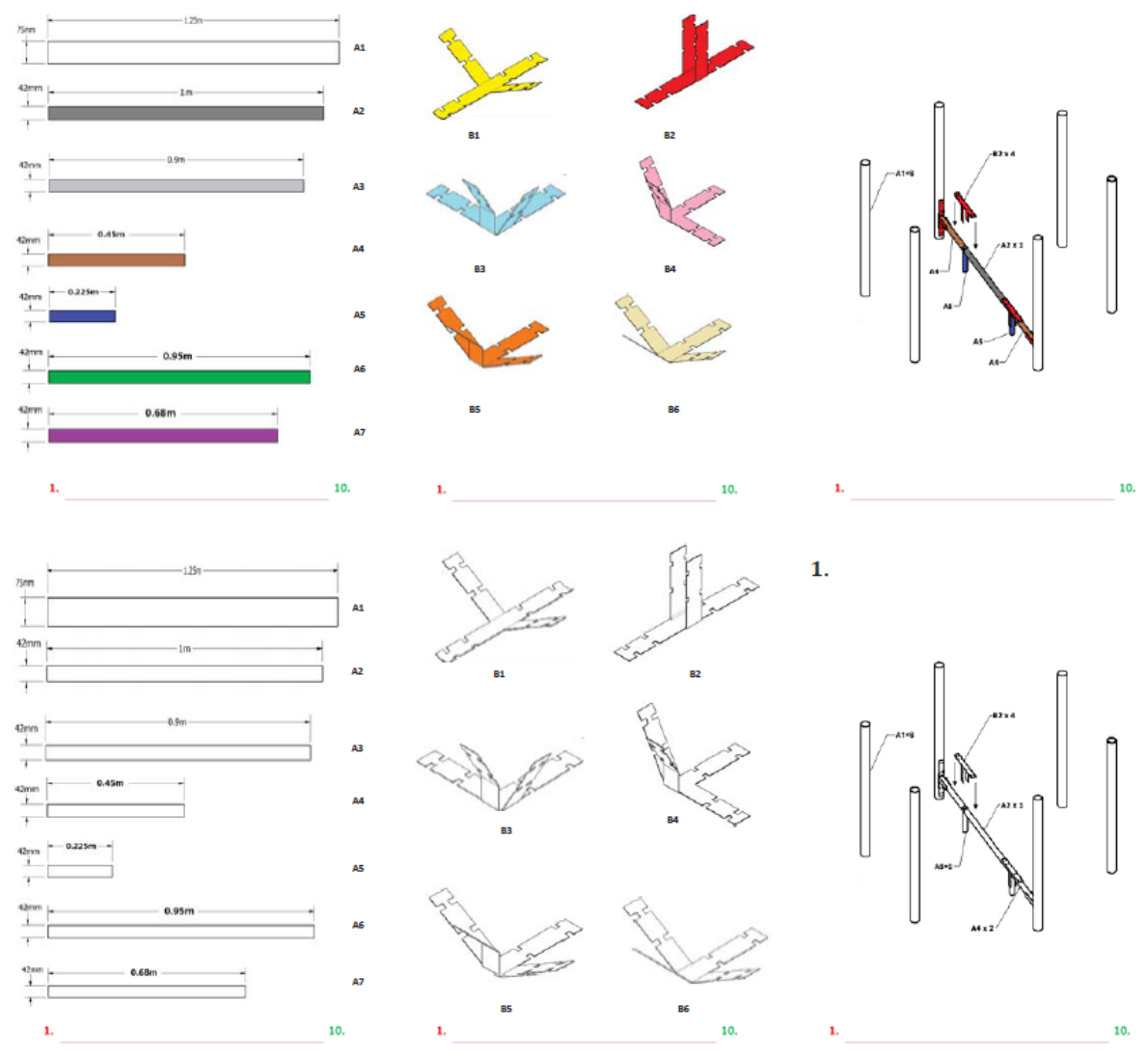

Figure 7: Exemplar of color-coded instructions (top), which was favorited, and black and white (bottom)

Three groups were tested. One of them was provided a colored set of instructions and the other two groups a black and white line version. The former one welcomed the aid provided by the colors to identify parts and to place them correctly in the assembly. They suggested the use of a more marked contrast between colors (e.g., blue and red instead of orange and red). The other groups made the unsolicited suggestion of including colors in the diagrams. All groups indicated the need for labelling the components (bamboo poles and metal connections) to simplify their identification as parts and their use at each step of the assembly. 
In the tests carried out by these three groups the hypothesis for preference towards subassemblies remained valid. Further grouping of parts (i.e., these comprising the body of the hut and the roof) were favored. In practical terms this translates to instructions in which the diagrams that refer to the construction of the body are preceded by the inventory of its components only and then, clearly separated, the components and assembly steps of the roof (i.e., parts of the body of the hut followed by the instructions to build the body and subsequently parts of the roof followed by the instructions to build the roof). This approach decreased time at the tasks, increased clarity and contrasts with the strategy of presenting an overall inventory of parts and the very start of the assembly. Segmentation (i.e., detail steps within the sequence of a single operation) was well received and various examples of this approach (e.g., magnification of details, correct vs incorrect comparisons) were considered for the subsequent iteration.

The second iteration for the optimization of the instructions yielded results that highlighted specific ‘trouble steps' in the assembly. These were identified as: (1) assembly of the lower plane of the hut body. These operations and tasks are framed with a thick solid line in Figure 8 to allow a comparative study amongst teams; (2) orientation and fastening of the body-roof connections, framed with a double line in Figure 8; and (3) positioning and joining body and roof subassemblies, framed with a broken line in Figure 8.

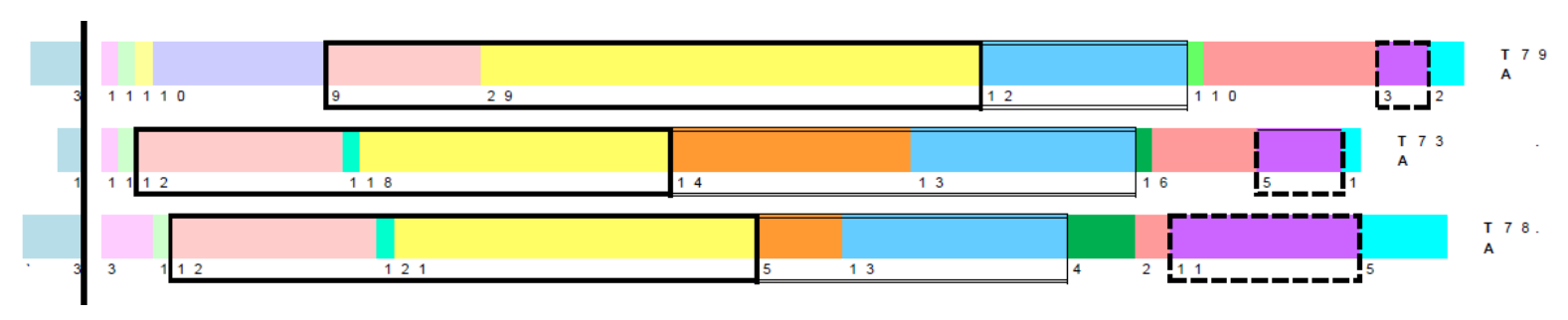

Figure 8: Timings on operations for the three groups in the second iteration

Recommendations expressed in the post-exercise interviews and on the scoring sheets, along with best practices for increasing effectiveness of the participants and decreasing difficulty ratings (Miller, 1956; Richardson et al., 2004; Schumacher, 2013) , informed the design of new illustrations for the subsequent iteration. The next section summarises how these changes were implemented and made an impact on the participants’ performance. 


\section{Third Iteration}

The third iteration in the diagnostic testing took place within the framework of skillset relevance. Individuals with no formal training in 3D representational conventions and with no technical background participated in this stage. They were graduates of Asian and Indian nationality and incidentally there was a large proportion of females (70\%) amongst the teams. Six groups were tested in this iteration. The participants conducted the assemblies with instructions that contained strategies to decrease difficulty rating, as suggested by different scholars cited below. Figure 9 presents three examples. The comprehensive list is as follows:

(v) Labelling parts and numbering steps. Participants of the first two iterations experienced problems when identifying the parts. Assembly instructions comprehension improved when labelling was introduced as well as grouping of the components per sub-assembly and organising them according to the order of the assembly. This was in agreement with widely reported literature (Madan et al., 1995; Richardson et al., 2004; Spinillo \& Dyson, 2001) which stems from Miller's 'Chunking Theory’ (Miller, 1956) which explains how cognitive load is reduced by the use of these resources.

(vi) 'Do this not this' (i.e. correct vs incorrect action) was used as reported in (Schumacher, 2013) to enrich assembly steps of the assembly, in particular those of the lower plane of the hut body. This strategy did not show improvements in this new iteration. The reasons are behind the nature of the task, heavy on intrinsic complexity. This aspect will be discussed later in this manuscript.

(vii) 'Zoom in'. This resource, also reported in (Schumacher, 2013) was used to magnify details of the assembly action around the lower plane of the hut body and also of the positioning and joining the roof and body sub-assemblies. It was used with care to avoid confusion those participants with no formal 3D visualization training. Overall, the magnified details were perceived as a valuable improvement by all groups regardless of their technical training.

(viii) Reduce redundancy. Expendable details on the instructions (e.g., positioning of the roof subassembly onto the body aided by one person stepping into the hut, tightness level and positioning of the fasteners) were removed after observing that those steps were of low relevance to participants. An intended absence of detail (Schumacher, 2013) can have positive impact on the 
empowerment and engagement of the participants, as their abilities are invited to contribute to the activity (Fuglesang, 1973).

(ix) Auditing. Generally participants were observed to search for visual cues that would indicate correct progress for the assembly. Explicit 'check points' were introduced for the 'trouble steps' using an 'Action/State' strategy (Schumacher, 2013). This proved useful to all participating groups. This taskbased approach was an indication of the participants' engagement on the task (Eiriksdottir, 2011)

(x) Viewing angle, which was kept fixed throughout the whole sequence for the hut body and roof (Heiser et al., 2004; Schumacher, 2013). However, as the positioning of the body-roof connections scored high in difficulty rating, this was resolved by introducing a change of angle which showed the task from a top view. Despite this resource being contrary to the general rule of keeping stable points of view and realistic arrangements, it proved more useful than orientation details added on the standard plan view of the assembly.

(xi) Symmetry became a complex resource and its use in this activity did not yield conclusive results. Explicit diagrams showing symmetry for the roof subassembly and its positioning over the body were considered redundant and such repetitive operations were skipped from the instructions and presented only once (Agrawala et al., 2003). However the same groups requested explicit symmetry diagrams for the assembly of the top and lower planes of the hut body. Results showed no relevance to skillset or cultural backgrounds.
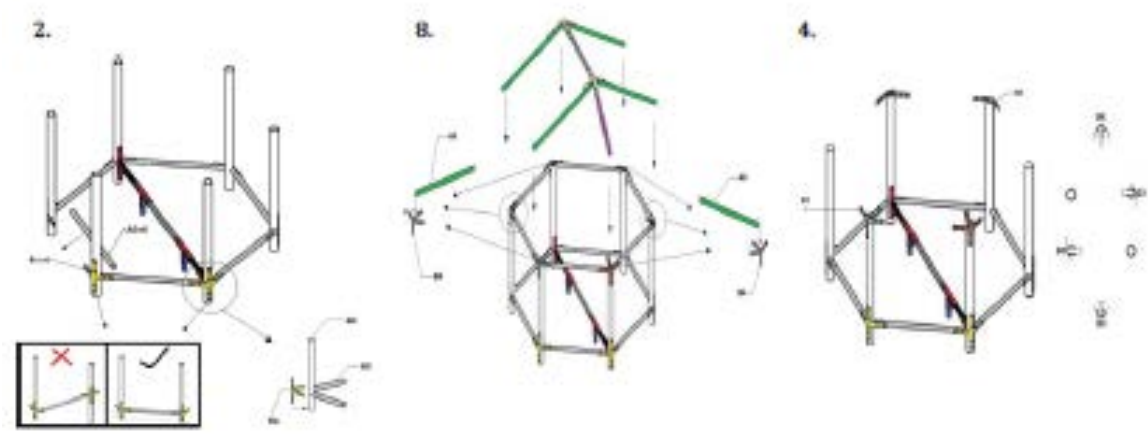

Figure 9: Exemplars of third iteration: (l-r) 'Do this not this' example and 'Zoom in', Symmetry, change of Viewing angle. The participants noted that these approaches enriched the depictions 


\section{Addressing intrinsic complexity}

The difficulty rating of the orientation and fastening of the body-roof connections and that of the positioning and joining body and roof subassemblies were reduced by employing the aforementioned strategies. However, the assembly of the lower plane of the hut body remained a challenge to the teams in the latter iterations. The tasks of this operation took an average of $46.1 \%$ (std 2.3) of the total activity time (bold line in Figure 10, pink and green boxes grouped).

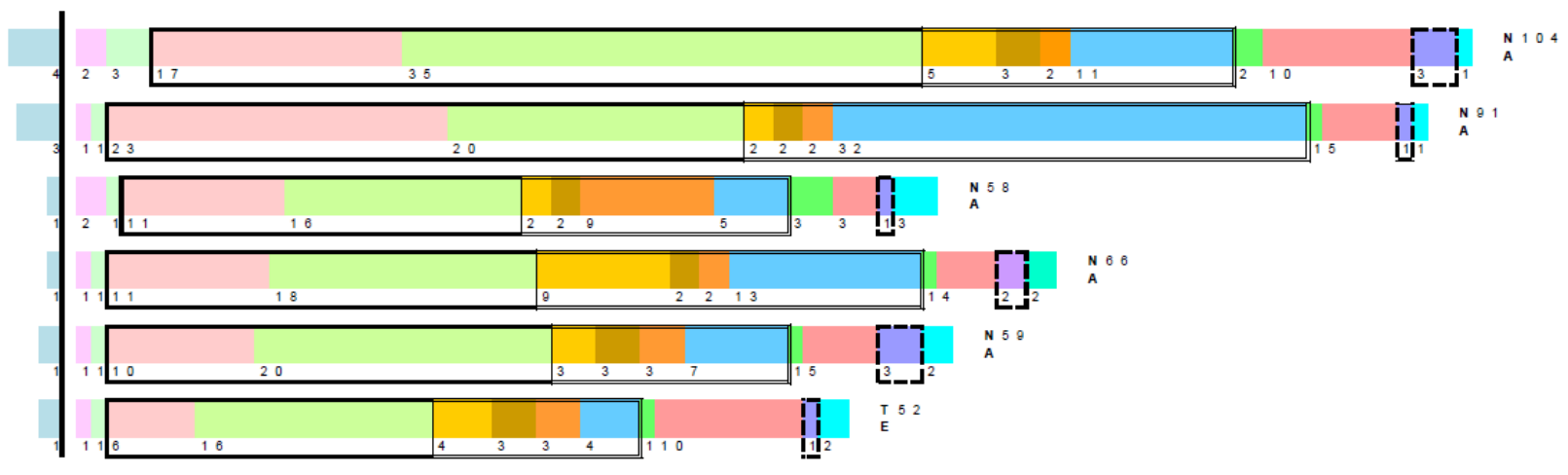

Figure 10: Timings on operations for the six groups in the third iteration

We hypothesized that the sequential steps for this operation contained heavy intrinsic complexity (i.e., when the operation cannot be further segmented and require of the participants' own ability to interpret and execute successfully). For example, if symmetry was not detected by the participants in this operation, the six horizontal beams that formed the hexagonal base of the body and the connections to the vertical poles presented an operation of twelve units, versus only six if symmetry was spotted. This elevated the number of tasks and posed an impact on cognitive load for the end-user. It was not possible to establish with any level of confidence whether the assumption of symmetry in the structure (i.e., to save repetitive steps that disengaged the readers (Richardson et al., 2004)) was aligned to the intention of empowerment of the end-user (Fuglesang, 1973). This finding is in agreement with reported literature: intrinsic complexity and inherent cognitive load are imposed by the basic characteristics of the object, rather than by the instruction in the sequential step itself (Sweller, 1994). 


\section{Conclusions}

The planning and presentation of the bamboo hut assembly instructions aimed at the creation of a set of sequences easy to understand for the wider audience regardless of their degree of literacy; friendly and accessible with the use of pictorial representations and with an embedded 'troubleshooting' system to support the end-user through the 'as identified' most complex and cognitive-loaded operations and tasks. We conducted participant-centered testing in this study to systematically assess difficulty rating, evaluate operation complexity and measure completion time. Informed by previous studies and reported best practices, we introduced strategies that addressed these issues in a stepped manner through three iterations performed to yield an optimized set of instructions tested by a diverse population with regards to experience, assembly abilities and nationalities. This is relevant to instructions designers who wish to use diagnostic testing as a tool to promote and improve user understanding and interpretation of assembly instructions. This approach facilitates userproduct interaction and provides a channel for reaching out to broad audiences

The main learnings drawn from this study for the benefit of Instructions Design Practice and Research are the following:

- Color coding, numbering steps, grouping of parts ('chunking'), and segmentation of operations were perceived as friendly and accessible as well as time-efficient

- The completion time was shorter when subassemblies were presented

- Assembly depicting actions were preferred to static structural diagrams

- Subassembly inventories immediately preceding such operations were preferred to an overall initial inventory

- Self-auditing gateways (i.e., 'check points') for the complex operations, magnifications and segmentation of operations were received as empowering, increasing the level of engagement amongst the participants

- Segmentation of symmetrical operations and redundant details were perceived as patronizing

- Operations with a heavy intrinsic complexity benefit from strategies that are normally perceived as counter-productive for the clarity of the tasks (e.g., change of angle view, distortions and alterations in arrangements)

- Difficulties in deriving symmetry originated action steps with a large number of tasks to deal with in a single sequential step. This created a large load of inherent cognitive 
load that was disadvantageous to participants of little technical expertise or less capable with 3D visualizations

Despite the optimization of the instructions planning and presentation, this work has shown that some operations in the assembly of the hut remain intrinsically complex for the participants, regardless of their educational or cultural background. Further work prior to the field testing will focus on the study of these operations and their sequential presentation. A deeper understanding of the inherent cognitive load needs to be achieved in an attempt to reduce difficulty and accomplish the design of more user-friendly instructions.

Note: Images in figure 1 (top row and bottom left) are kindly provided by Ms Lara Lewington. Image (bottom right) is presented as it appears in (Drake et al., 2011)

\section{References}

Agrawala, M., Phan, D., Heiser, J., Haymaker, J., Klingner, J., Hanrahan, P., \& Tversky, B. (2003). Designing effective step-by-step assembly instructions. ACM Trans. Graph., 22(3), 828-837. doi: 10.1145/882262.882352

Bramorski, T., Madan, M., Motwani, J., \& Sundarraj, R. P. (2000). Improving competitiveness of ready - to - assemble manufacturers through information technology. Logistics Information Management, 13(4), 201-209. doi: 10.1108/09576050010340820

Chandler, C. I. R., Meta, J., Ponzo, C., Nasuwa, F., Kessy, J., Mbakilwa, H., . . . Reyburn, H. (2014). The development of effective behaviour change interventions to support the use of malaria rapid diagnostic tests by Tanzanian clinicians. Implementation Science, 9, 83-83. doi: 10.1186/1748-5908-9-83

Chung, K. F., \& Yu, W. K. (2002). Mechanical properties of structural bamboo for bamboo scaffoldings. Engineering Structures, 24(4), 429-442. doi: 10.1016/S0141-0296(01)00110-9

Clark, R. C., Nguyen, F., \& Sweller, J. (2011). Efficiency in Learning: Evidence-Based Guidelines to Manage Cognitive Load: Wiley.

Drake, R., Morton, R., \& Torres-Sanchez, C. (2011). Lessons from India: Bamboo as a solution for low-cost housing. Paper presented at the EWB-UK National Research Conference ‘Our Global Future’, London, UK. http://tinyurl.com/zyxsmxg 
Eiriksdottir, E. (2011). The role of principles in instructions for procedural tasks: timing of use, method of study, and procedural instruction specificity. (PhD), Georgia Institute of technology.

Fuglesang, A. (1973). Applied communication in developing countries: ideas and observations. Uppsala, Sweden: Dag Hammarskjöld Foundation.

Ganier, F. (2004). Factors affecting the processing of procedural instructions: implications for document design. Professional Communication, IEEE Transactions on, 47(1), 15-26. doi: 10.1109/TPC.2004.824289

Heiser, J., Phan, D., Agrawala, M., Tversky, B., \& Hanrahan, P. (2004). Identification and validation of cognitive design principles for automated generation of assembly instructions. Paper presented at the Proceedings of the working conference on Advanced visual interfaces, Gallipoli, Italy.

Heiser, J., \& Tversky, B. (2006). Arrows in Comprehending and Producing Mechanical Diagrams. Cognitive Science, 30(3), 581-592. doi: 10.1207/s15516709cog0000_70

Houts, P. S., Doak, C. C., Doak, L. G., \& Loscalzo, M. J. (2006). The role of pictures in improving health communication: A review of research on attention, comprehension, recall, and adherence. Patient Education and Counseling, 61(2), 173-190. doi:

dx.doi.org/10.1016/j.pec.2005.05.004

Hsieh, M. H., Huang, C. Y., Luh, D. B., Liu, S. F., \& Ma, C. H. (2013). An application of implementing a cognitive structure model to obtain consensus from consumers. International Journal of Design, 7(2), 53-65.

Janssen, J. J. A. (1995). Building with bamboo: a handbook (2nd ed. ed.). United Kingdom: ITDG Publishing.

Jonassen, D. H., Tessmer, M., \& Hannum, W. H. (1999). Task Analysis Methods for Instructional Design: Lawrence Erlbaum Associates.

Kim, S., Woo, I., Maciejewski, R., Ebert, D. S., Ropp, T. D., \& Thomas, K. (2010). Evaluating the effectiveness of visualization techniques for schematic diagrams in maintenance tasks. Paper presented at the Proceedings of the 7th Symposium on Applied Perception in Graphics and Visualization, Los Angeles, California.

Leake, G., Toole, K., Divis, P., \& Torres-Sanchez, C. (2010). Bamboo as a solution for lowcost housing and storage in Pabal (India). Paper presented at the EWB-UK National Research Conference' From Small Steps to Giant Leaps: putting reseach into practice', London, UK. http://tinyurl.com/zdvunu4 
Likert, R. (1932). A technique for the measurement of attitudes. Archives of psychology, 22(140), 1-55.

Lopes Peres, R., \& Barreto Campello, S. (2014). In the way of arrows: the production of illustrated instruction for children and its possibilities of interpretation. Paper presented at the 6th Information Design International Conference, 5th InfoDesign, 6th CONGIC, São Paulo, Brazil.

Madan, M., Bramorski, T., \& Sundarraj, R. P. (1995). The effects of grouping parts of ready - to - assemble products on assembly time: an experimental study. International Journal of Operations \& Production Management, 15(3), 39-49. doi:

$10.1108 / 01443579510080544$

Miller, G. A. (1956). The magical number seven, plus or minus two: some limits on our capacity for processing information. Psychological Review, 63(2), 81-97. doi: $10.1037 / \mathrm{h} 0043158$

Minke, G. (2012). Building with Bamboo: Design and Technology of a Sustainable Architecture: Birkhäuser.

Mishra, M., Behera, S. K., Majumdar, S., \& Kharagpur, D. (2009). Comparison of cost of dwelling units using bamboo as reinforcing material. Paper presented at the 7th All India People's Technology Congress, Shelter sub-congress, Science City, Kolkata.

Richardson, M., Jones, G., \& Torrance, M. (2004). Identifying the task variables that influence perceived object assembly complexity. Ergonomics, 47(9), 945-964. doi: 10.1080/00140130410001686339

Rosling, H., Rosling, R. A., \& Rosling, O. (2005). New software brings statistics beyond the eye. Statistics, Knowledge and Policy: Key Indicators to Inform Decision Making. Paris, France: OECD Publishing, 522-530.

Schumacher, P. (2007). Creating effective illustrations for pictorial assembly instructions. Information Design Journal, 15(2), 97-109. doi: 10.1075/idj.15.2.01sch

Schumacher, P. (2009). Pictorial Communication in Developing Countries: A Literature Review. Visual: Design: Scholarship Research Journal of the Australian Graphic Design, 4(1), 41-68 1833-2226. doi: oai:ura.unisa.edu.au:53524

Schumacher, P. (2013). A Pattern Language for Pictorial Assembly Instructions (PAIs). Information Design Journal, 20(2), 111-135. doi: 10.1075/idj.20.2.03sch

Shepherd, A. (1998). HTA as a framework for task analysis. Ergonomics, 41(11), 1537-1552. doi: 10.1080/001401398186063 
Söderberg, C., Johansson, A., \& Mattsson, S. (2014). Design of Simple Guidelines to Improve Assembly Instructions and Operator Performance. Paper presented at the The sixth Swedish Production Symposium, Gothenburg, Sweden.

Spinillo, C. G., \& Dyson, M. C. (2001). An exploratory study of reading procedural pictorial sequences. Information Design Journal, 10(2), 154-168.

Stanton, N. A. (2006). Hierarchical task analysis: Developments, applications, and extensions. Applied Ergonomics, 37(1), 55-79. doi: dx.doi.org/10.1016/j.apergo.2005.06.003

Sweller, J. (1994). Cognitive load theory, learning difficulty, and instructional design. Learning and Instruction, 4(4), 295-312. doi: dx.doi.org/10.1016/0959-4752(94)90003-5

Viola, I., \& Groeller, M. E. (2005). Smart visibility in visualization. Paper presented at the Proceedings of the First Eurographics conference on Computational Aesthetics in Graphics, Visualization and Imaging, Girona, Spain. http://diglib.eg.org/handle/10.2312/COMPAESTH.COMPAESTH05.209-216 